\title{
A Case of Rapidly Growing Extraocular Sebaceous Carcinoma
}

\author{
Joon Ho Lee ${ }^{1}$, \\ Hea-Kyeong Shin ${ }^{1}$, \\ Tae Jung Jang ${ }^{2}$ \\ Departments of ${ }^{I}$ Plastic and Reconstructive \\ Surgery and ${ }^{2}$ Pathology, Dongguk University \\ College of Medicine, Gyeongju, Korea
}

No potential conflict of interest relevant to this article was reported.

\begin{abstract}
Sebaceous carcinoma is a rare malignant tumor differentiated from the adnexal epithelium of sebaceous glands and forms less than $1 \%$ of all cutaneous malignancies. We present a case of a 93-year-old woman with a rapidly growing mass on the right cheek. Initial histiopathologic finding was basal cell carcinoma. The mass was widely excised and superficial parotidectomy was performed while preserving the facial nerve branches. The resulting defect was covered with a transposition flap from the ipsilateral posterior auricular area and the donor site was closed primarily. However, histopathologic examination of the excised mass showed a poorly differentiated sebaceous carcinoma with a clear resection margin. The diagnosis of sebaceous carcinoma can be difficult to make at initial presentation. This report describes a rare case of a rapidly growing extraocular sebaceous carcinoma, which resulted in a good treatment outcome, and provides a review of relevant literature.
\end{abstract}

Keywords: Sebaceous adenocarcinoma / Basal cell carcinoma

\section{INTRODUCTION}

Sebaceous carcinoma is a rare malignant tumor differentiated from the adnexal epithelium of sebaceous glands and forms less than $1 \%$ of all cutaneous malignancies. It is estimated that approximately $25 \%$ of all reported cases of sebaceous carcinoma occur in extraocular site. It has been reported that more than $70 \%$ of sebaceous carcinomas are located in the head and neck regions, where sebaceous glands are most commonly located [1,2]. This is a report of our experience of a rapidly growing sebaceous carcinoma on the right cheek of an elderly woman.

\section{CASE REPORT}

A 93-year-old woman visited our hospital with a chief complaint

Correspondence: Hea-Kyeong Shin

Department of Plastic and Reconstructive Surgery, Dongguk University College of Medicine, 87 Dongdae-ro, Gyeongju 780-350, Korea

E-mail: shinheakyeong@hanmail.net

Received January 28, 2014 / Revised February 27, 2014 / Accepted March 5, 2014 of palpable mass over the right cheek. The mass began to appear about 1 year ago and was gradually enlarged (Fig. 1). On physical examination, a round, $2.0 \times 1.5 \mathrm{~cm}$ sized erythematous mass was observed on her right cheek. The patient complained of a slight itching sense, but neither pain nor tenderness was observed. The patient had no specific underlying disease, no history of operation, no history of radiation therapy and no familial history of cancer.

Punch biopsy was performed at the dermatology department and the pathological findings confirmed the diagnosis of basal cell carcinoma. The patient and her family were hesitant to undergo surgery because of her old age. About 7 months later, the patient revisited us for surgery with a significantly enlarged $5.5 \times 4.5 \times$ $4.0 \mathrm{~cm}$ sized mass (Fig. 2). It was confirmed that the mass began to grow rapidly about 2 weeks ago without trauma or any specific medical history. It showed focal ulceration with yellowish purulent discharge. The patient suffered from pain, and mild tenderness was also observed. However, there was no localized or generalized lymphadenopathy and her facial nerve function was intact. 


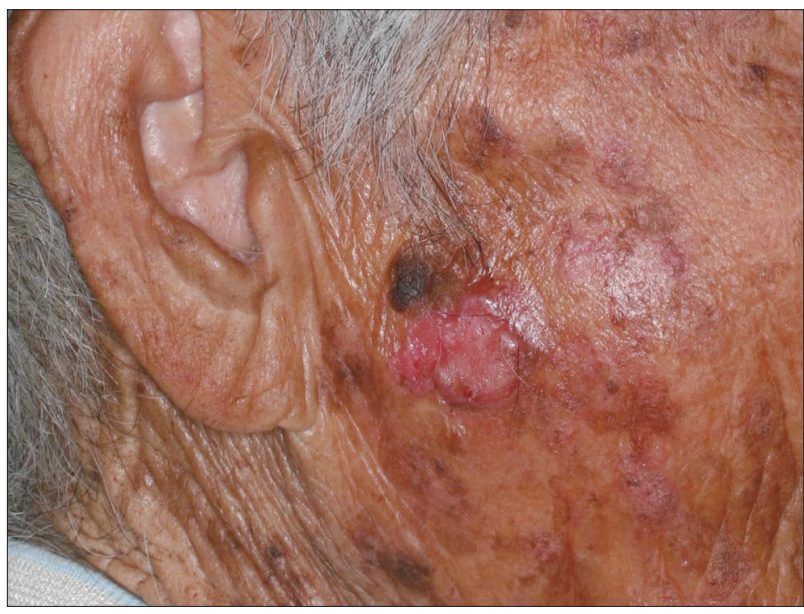

Fig. 1. An erythematous mass on the right cheek.

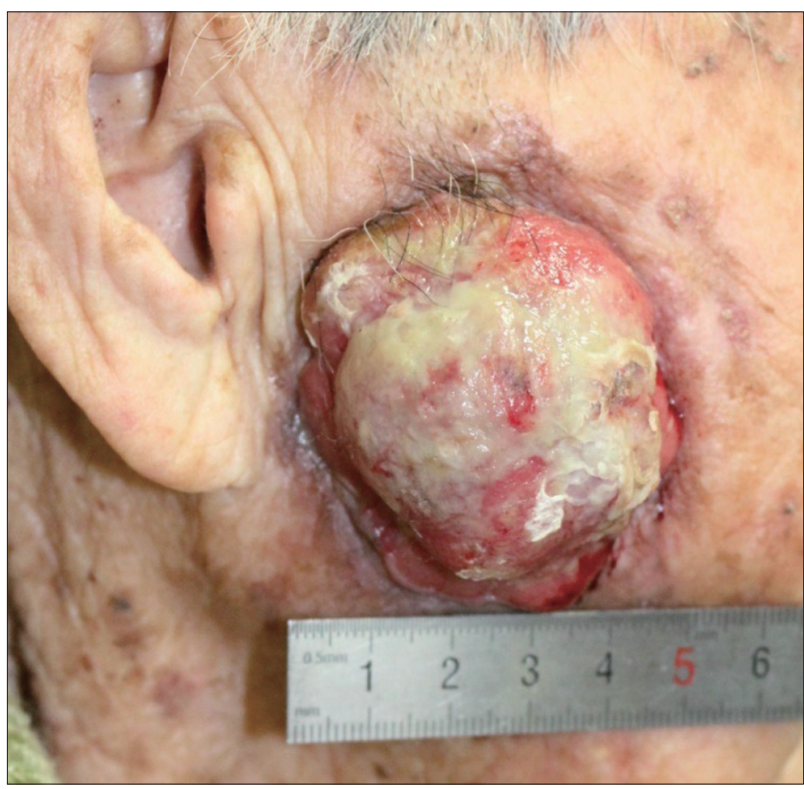

Fig. 2. Enlarged mass with focal ulceration and yellowish discharge, 7 months after the first visit.

We recommended other radiologic examinations to identify the degree of tumor invasion and metastasis, but those could not be progressed because the patient and her family wanted only a palliative treatment.

Under general anesthesia, the mass was widely excised with a safety margin of $5 \mathrm{~mm}$, and superficial parotidectomy was performed because of tumor invasion to the parotid gland (Fig. 3). Based on the result of frozen biopsy, the resection margin was clear, so that we could preserve the facial nerve branches (Fig. 4).

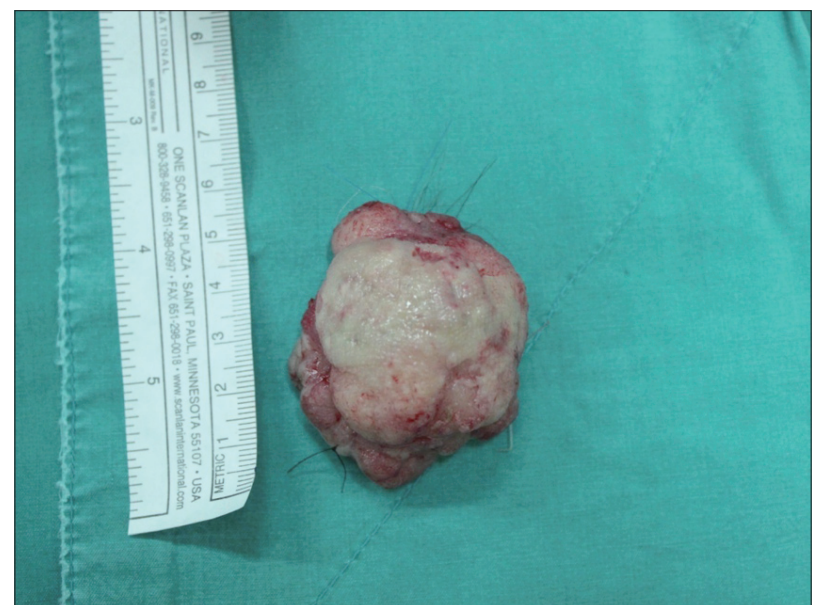

Fig. 3. Excised mass measuring $5.5 \times 4.5 \times 4.0 \mathrm{~cm}$ in size.

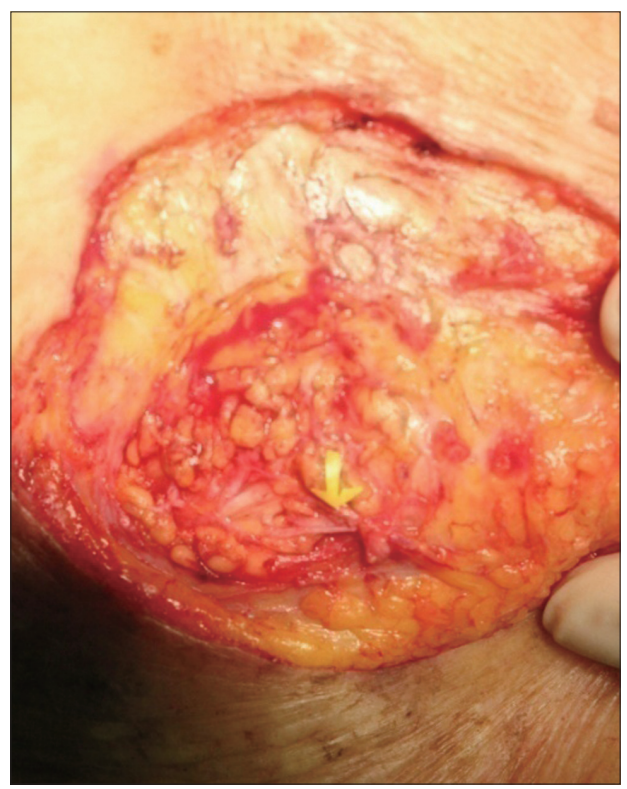

Fig. 4. Resulting defect after wide excision. Note the preserved facial nerve branch (yellow arrow).

The resulting defect was covered with a transposition flap from the ipsilateral posterior auricular area (Fig. 5) and the donor site was closed primarily (Fig. 6).

A histopathologic examination of the excised mass showed a poorly differentiated sebaceous carcinoma with a clear resection margin (Fig. 7). The patient was followed up 10 months postoperatively and the long-term result was satisfactory without any complications (Fig. 8). 


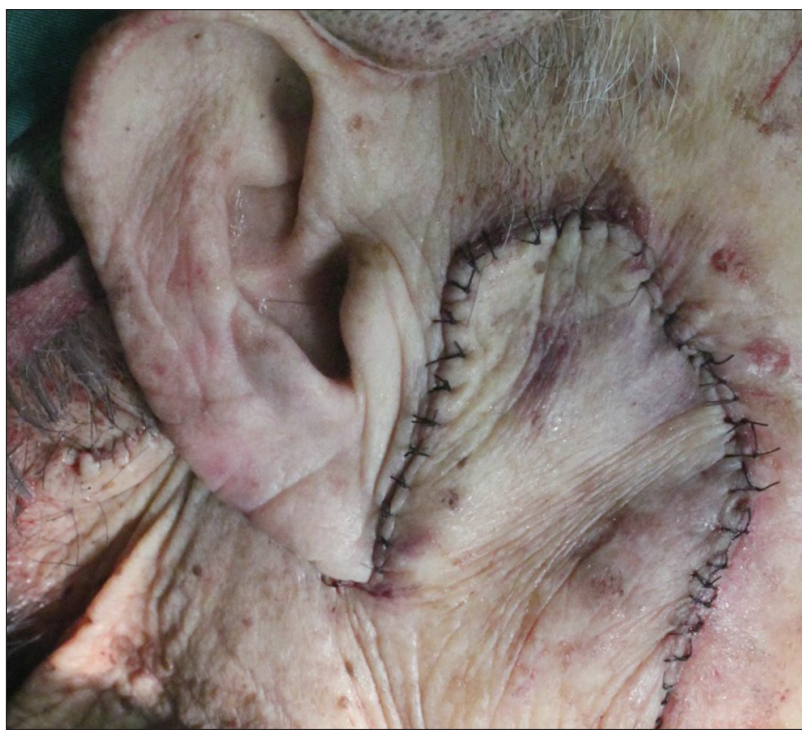

Fig. 5. Immediate postoperative appearance showing the defect covered with a transposition flap.

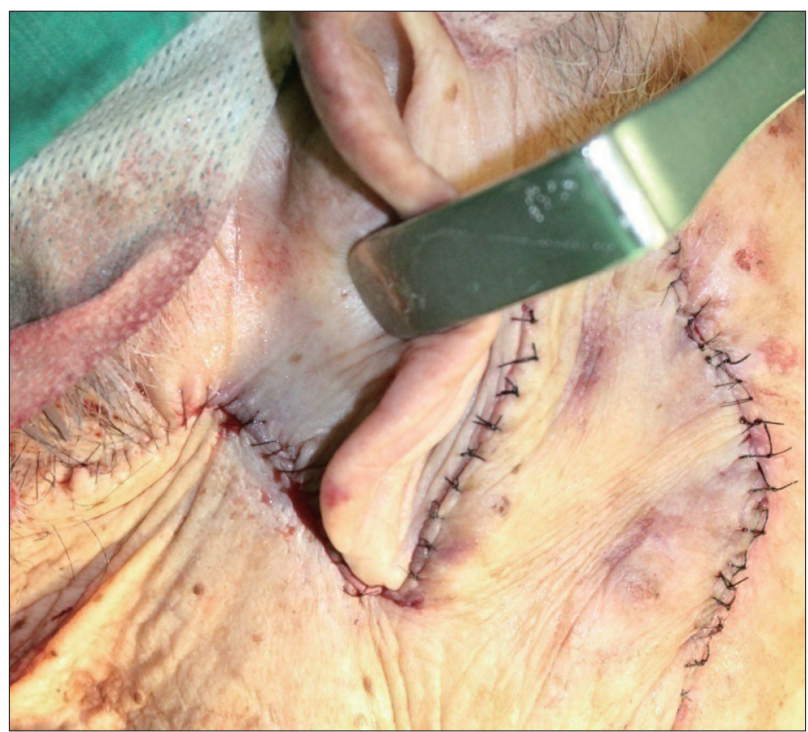

Fig. 6. Immediate postoperative appearance showing primary closure of the donor site.

\section{DISCUSSION}

A tumor of sebaceous glands usually starts as a solitary, erythematous or often pale yellow-colored, firm-to-hard, slowly growing nodule mainly in the head and neck regions. It is a rare malignant neoplasm comprising less than $1 \%$ of all cutaneous malignancies. The most common site for sebaceous carcinoma is the eyelids. Reported ocular sebaceous carcinoma was approximately three-

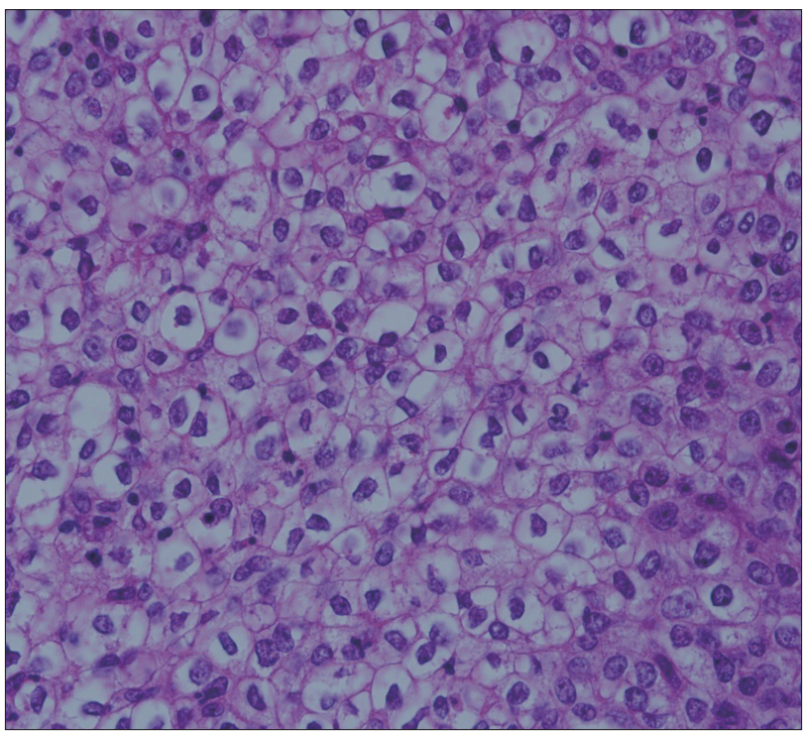

Fig. 7. Photomicrograph showing undifferentiated cells and large cells with vesicular and ill defined bubbly cytoplasm $(\mathrm{H} \& \mathrm{E}, \times 200)$.

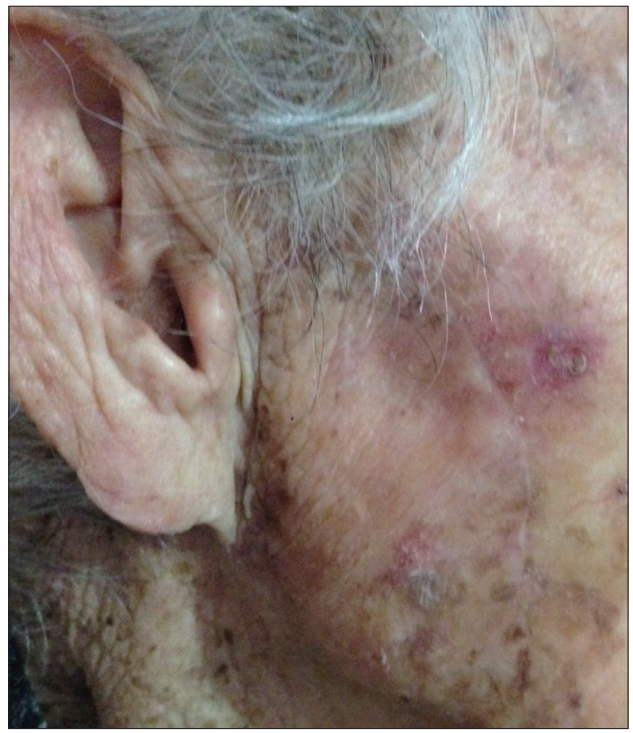

Fig. 8. Postoperative 10 months view. The wound was healed well without any complication.

times more common than the extraocular sebaceous carcinoma [1-3]. However, Dasgupta et al. [4] reported that $38.7 \%$ of sebaceous carcinomas occurred on eyelids. Dores et al. [5] reported that $45.9 \%$ of sebaceous carcinoma occurred on eyelids. This difference in the reported proportion was not only because of population-based studies of sebaceous carcinoma that have been infrequent, but also due to a small number of cases studied [5].

In general, extraocular sebaceous carcinoma is less aggressive 
than ocular sebaceous carcinoma [4]. However, some authors have reported that extraocular tumors preferentially show a rapid growth. This is especially true in elderly women [6]. In the present patient, the lesion was rapidly growing in just 2 weeks.

Sebaceous carcinoma may be associated with Muir-Torre syndrome, defined as an autosomal dominant genetic trait, and the presence of one or more sebaceous tumors (adenoma, epithelioma, or carcinoma) in association with at least one visceral malignancy. It is shown related to germ line mutations in the $\mathrm{MSH} 2$ and MLH1 genes found on chromosomes $3 \mathrm{p}$ and $2 \mathrm{p}$, respectively [5]. In this present patient, however, it is less likely to be Muir-Torre syndrome considering her age, nonspecific familial cancer history, and symptoms that are devoid of a visceral tumor.

The primary treatment of sebaceous carcinoma is a wide excision and frozen section with a safety margin of 5 to $6 \mathrm{~mm}$. Radiotherapy or chemotherapy has been the treatment of choice with postoperative care particularly when metastatis is confirmed. However, general guideline for radiotherapy and chemotherapy has not been established in this regard.

The diagnosis of sebaceous carcinoma can be difficult to make at initial presentation. Well-differentiated sebaceous carcinomas are not difficult to make a diagnosis because tumor cells have obvious cytoplasmic lipid vesicles that are characteristic of sebaceous carcinoma. But, poorly differentiated sebaceous carcinomas lack lipid vesicles and consist of predominantly basaloid tumor cells that imitate carcinomas such as basal cell carcinoma and squamous cell carcinoma [1]. Moreover, the punch biopsy only represents a small part of the lesion. It might result in a false-negative diagnosis or in missing a more severe part of the lesion. The present patient had undergone punch biopsy initially, and the re- sult confirmed a basal cell carcinoma. However, in contrast to basal cell carcinoma, sebaceous carcinoma can be an aggressive form of cancer. Local recurrence rate after surgical excision within 5 years is found to be approximately $9 \%$ to $36 \%$ in sebaceous carcinoma and $10.1 \%$ in basal cell carcinoma. Furthermore, metastasis occurs at relatively higher rate than that observed basal cell carcinoma $[1,7]$. The present patient wanted only a palliative treatment so that we did not perform repetitive histological examination before the operation. Therefore, although in rare cases, if diagnosed basal cell carcinoma grows rapidly, proper evaluation is essential to assess whether sebaceous carcinoma was misdiagnosed.

This report describes a rare case of a rapidly growing extraocular sebaceous carcinoma, which resulted in a good treatment outcome of cosmetic and functional aspect, and provides a review of relevant literature.

\section{REFERENCES}

1. Nelson BR, Hamlet KR, Gillard M, Railan D, Johnson TM. Sebaceous carcinoma. J Am Acad Dermatol 1995;33:1-15.

2. Shields JA, Demirci H, Marr BP, Eagle RC Jr, Shields CL. Sebaceous carcinoma of the ocular region: a review. Surv Ophthalmol 2005;50:103-22.

3. Urban FH, Winkelmann RK. Sebaceous malignancy. Arch Dermatol 1961;84:63-72.

4. Dasgupta T, Wilson LD, Yu JB. A retrospective review of 1349 cases of sebaceous carcinoma. Cancer 2009;115:158-65.

5. Dores GM, Curtis RE, Toro JR, Devesa SS, Fraumeni JF Jr. Incidence of cutaneous sebaceous carcinoma and risk of associated neoplasms: insight into Muir-Torre syndrome. Cancer 2008;113:3372-81.

6. King DT, Hirose FM, Gurevitch AW. Sebaceous carcinoma of the skin with visceral metastases. Arch Dermatol 1979;115:862-3.

7. Levi F, Randimbison L, Maspoli M, Te VC, La Vecchia C. High incidence of second basal cell skin cancers. Int J Cancer 2006;119:1505-7. 